\title{
Contents $^{1}$
}

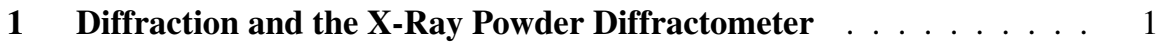

1.1 Diffraction . . . . . . . . . . . . . . . . . 1

1.1.1 Introduction to Diffraction . . . . . . . . . . . 1

1.1 .2 Bragg's Law . . . . . . . . . . . . . . . . . 3

1.1.3 Strain Effects . . . . . . . . . . . . . . . . . 6

1.1 .4 Size Effects . . . . . . . . . . . . . . . . . . 6

1.1.5 A Symmetry Consideration . . . . . . . . . . . . . 8

1.1.6 Momentum and Energy . . . . . . . . . . . . . . . . . 9

1.1 .7 Experimental Methods . . . . . . . . . . . . . . . . 10

1.2 The Creation of X-Rays . . . . . . . . . . . . . . . . . . . 12

1.2.1 Bremsstrahlung . . . . . . . . . . . . . . . . 13

1.2.2 Characteristic Radiation . . . . . . . . . . . . . . 15

1.2.3 Synchrotron Radiation . . . . . . . . . . . . . . . 20

1.3 The X-Ray Powder Diffractometer . . . . . . . . . . . . . . . 22

1.3.1 Practice of X-Ray Generation . . . . . . . . . . . 22

1.3.2 Goniometer for Powder Diffraction . . . . . . . . . . . . 24

1.3.3 Monochromators, Filters, Mirrors . . . . . . . . . . 27

1.4 X-Ray Detectors for XRD and TEM . . . . . . . . . . . . . . . . 29

1.4.1 Detector Principles . . . . . . . . . . . . . . . . . . . . . . . 29

1.4.2 Solid State Detectors . . . . . . . . . . . . . . . . . 31

1.4.3 Position-Sensitive Detectors . . . . . . . . . . . . 33

1.4.4 Charge Sensitive Preamplifier . . . . . . . . . . . . . 34

1.4.5 Other Electronics . . . . . . . . . . . . . . . . . . . . 35

1.5 Experimental X-Ray Powder Diffraction Data . . . . . . . . . 37

1.5.1 * Intensities of Powder Diffraction Peaks . . . . . . . 37

1.5.2 Normals of Diffracting Planes . . . . . . . . . . . . 37

1.5 .3 Slit Width . . . . . . . . . . . . . . . . . . . . 38

1.5.4 Lorentz Factor . . . . . . . . . . . . . . 38

\footnotetext{
${ }^{1}$ In section titles, the asterisk, “*, denotes a more specialized topic. The double dagger, “ $\ddagger$," warns of a higher level of mathematics, physics, or crystallography.
} 


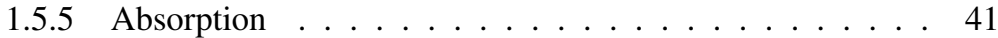

1.5 .6 Polarization . . . . . . . . . . . . . . . . 42

1.5.7 Multiplicity and Density . . . . . . . . . . . . . . . 42

1.5.8 Measured Intensities . . . . . . . . . . . . . . . . . . 43

1.5.9 Phase Fraction Measurement . . . . . . . . . . . . . . 44

1.5.10 Peak Ratio Method . . . . . . . . . . . . . . . . . . 44

1.5.11 Absorption Factors . . . . . . . . . . . . . . . 45

1.5.12 Example: Retained Austenite in Steels . . . . . . . . . . 46

1.5.13 Lattice Parameter Measurement . . . . . . . . . . . . 48

1.5.14 * Refinement Methods for Powder Diffraction Data . . . . 49

1.6 Further Reading . . . . . . . . . . . . . . . . . . . . . . . 52

1.7 Problems .................... 53

2 The TEM and Its Optics . . . . . . . . . . . . . . . . . . . . . . 59

2.1 Introduction to the Transmission Electron Microscope . . . . . . . 59

2.2 Working with Lenses and Ray Diagrams . . . . . . . . . . . . 63

$2.2 .1 \quad$ Single Lenses . . . . . . . . . . . . . . . . . . . . . 63

2.2 .2 Multi-Lens Systems . . . . . . . . . . . . . . . . . . . 67

2.3 Modes of Operation of a TEM . . . . . . . . . . . 68

2.3.1 Dark-Field and Bright-Field Imaging . . . . . . . . . . . 68

2.3.2 Selected Area Diffraction . . . . . . . . . . . . . . . . 73

2.3.3 Convergent-Beam Electron Diffraction . . . . . . . . . 77

2.3.4 Nanobeam Diffraction . . . . . . . . . . . . . . . . 78

2.3 .5 High-Resolution Imaging . . . . . . . . . . . . . . . 79

2.4 Practical TEM Optics . . . . . . . . . . . . . . . . . . . 82

2.4 .1 Electron Guns . . . . . . . . . . . . . . . . . . 82

2.4.2 Illumination Lens Systems _ . . . . . . . . . . . . . 85

2.4 .3 Imaging Lens Systems $\ldots \ldots \ldots$

2.5 Glass Lenses . . . . . . . . . . . . . . . . . . . . . . . . 88

2.5.1 Interfaces . . . . . . . . . . . . . . . . 88

2.5.2 Lenses and Rays . . . . . . . . . . . . . . . . . . . . 89

2.5.3 Lenses and Phase Shifts . . . . . . . . . . . . . . . . . . 92

2.6 Magnetic Lenses . . . . . . . . . . . . . . . . . . . . . . . 93

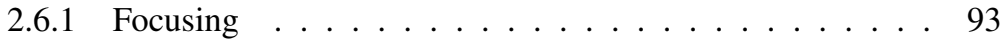

2.6 .2 Image Rotation . . . . . . . . . . . . . . . . . . . . 96

2.6 .3 Pole Piece Gap . . . . . . . . . . . . . . . . . . . . . . . 98

2.7 Lens Aberrations and Other Defects . . . . . . . . . . . . . 98

2.7.1 Spherical Aberration . . . . . . . . . . . . . . . . . . 98

2.7.2 Chromatic Aberration . . . . . . . . . . . . . . . . . . . . 99

2.7 .3 Diffraction . . . . . . . . . . . . . . . . 100

2.7 .4 Astigmatism . . . . . . . . . . . . . . . . . 101

2.7 .5 Gun Brightness . . . . . . . . . . . . . . . . . . 104

2.8 Resolution . . . . . . . . . . . . . . . . . . . . . . . 106

2.9 Further Reading . . . . . . . . . . . . . . . . . . . . . . . 108

2.10 Problems . . . . . . . . . . . . . . . . . . 109 
3 Neutron Scattering . . . . . . . . . . . . . . . . . 117

3.1 Neutrons and Neutron Scattering . . . . . . . . . . . . 117

3.1 .1 Neutron Scattering . . . . . . . . . . . . . . . 117

3.1.2 Properties of Neutrons . . . . . . . . . . . . . . . 118

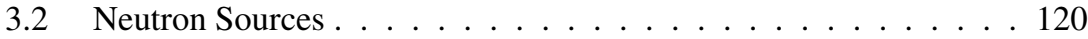

3.2 .1 Fission and Spallation . . . . . . . . . . . . . . . 120

3.2 .2 Moderation . . . . . . . . . . . . . . . . . . . . 121

3.3 Neutron Powder Diffractometers . . . . . . . . . . . . 122

3.3.1 Reactor-Based Powder Diffractometer . . . . . . . . . . . 122

3.3.2 Pulsed-Source-Based Powder Diffractometer . . . . . . . 122

3.4 Waves of Phase . . . . . . . . . . . . . . . 126

3.4.1 Phase in Elastic Scattering . . . . . . . . . . . . . 126

3.4.2 * Phase in Inelastic Scattering . . . . . . . . . . . . . . 128

3.5 Instruments for Measuring Larger Structures . . . . . . . . . . . . 129

3.5.1 Small-Angle Scattering . . . . . . . . . . . . . . . . . . . 129

$3.5 .2 *$ Neutron Reflectivity . . . . . . . . . . . . . . . 130

$3.6 *$ Inelastic Scattering . . . . . . . . . . . . . . . . . . . . 133

3.6.1 * Triple-Axis Spectrometer . . . . . . . . . . . . 133

3.6.2 * Fermi Chopper Spectrometer . . . . . . . . . . . . . . . 134

3.6.3 * Other Inelastic Instruments . . . . . . . . . . . . . . 136

$3.7 *$ Quasielastic Scattering . . . . . . . . . . . . . . . . . . 137

$3.8 *$ Magnetic Scattering . . . . . . . . . . . . . . . . . . . . . . . . 139

3.9 Nuclear Scattering . . . . . . . . . . . . . . . . . . . . . . . . 140

3.10 Further Reading . . . . . . . . . . . . . . . . . . . . . . . 141

3.11 Problems . . . . . . . . . . . . . . . . . . . . . . . . 142

4 Scattering . . . . . . . . . . . . . . . . . . 145

4.1 Waves and Scattering . . . . . . . . . . . . . . 145

4.1.1 Wavefunctions . . . . . . . . . . . . . . 145

4.1.2 Coherent and Incoherent Scattering _ . . . . . . . . . 148

4.1 .3 Elastic and Inelastic Scattering . . . . . . . . . . . . . . . 149

4.1.4 Wave Amplitudes and Cross-Sections . . . . . . . . . . 150

4.2 X-Ray Scattering . . . . . . . . . . . . . . . . . . . 154

4.2.1 Electrodynamics of X-Ray Scattering . . . . . . . . . 154

$4.2 .2 *$ Inelastic Compton Scattering . . . . . . . . . . . . . 158

4.2.3 X-Ray Mass Attenuation Coefficients . . . . . . . . . 160

4.3 Coherent Elastic Scattering . . . . . . . . . . . . . . . . . 162

4.3.1 $\ddagger$ Born Approximation for Electrons . . . . . . . . . . . 162

4.3.2 Atomic Form Factors-Physical Picture . . . . . . . . . 167

4.3.3 $\ddagger$ Scattering of Electrons by Model Potentials . . . . . . 170

4.3.4 $\ddagger *$ Atomic Form Factors-General Formulation . . . . . 174

4.4 Further Reading . . . . . . . . . . . . . . . . . . . . . . . 178

4.5 Problems . . . . . . . . . . . . . . . 178

5 Inelastic Electron Scattering and Spectroscopy _ . . . . . . . . . 181

5.1 Inelastic Electron Scattering _ . . . . . . . . . . . . . . 181

5.2 Electron Energy-Loss Spectrometry (EELS) . . . . . . . . . 183 
5.2 .1 Instrumentation . . . . . . . . . . . . . . . . . 183

5.2.2 General Features of EELS Spectra . . . . . . . . . . . 185

$5.2 .3 *$ Fine Structure . . . . . . . . . . . . . . . . . . 186

5.3 Plasmon Excitations . . . . . . . . . . . . . . . . . 190

5.3.1 Plasmon Principles . . . . . . . . . . . . . . . . . . 190

5.3.2 * Plasmons and Specimen Thickness . . . . . . . . . . 192

5.4 Core Excitations . . . . . . . . . . . . . . . . . . . . . . . . . . . 194

5.4.1 Scattering Angles and Energies-Qualitative . . . . . . 194

5.4.2 ‡Inelastic Form Factor . . . . . . . . . . . . . . . . . 197

5.4.3 ** Double-Differential Cross-Section, $\mathrm{d}^{2} \sigma_{\text {in }} / \mathrm{d} \phi \mathrm{d} E \ldots 201$

5.4.4* Scattering Angles and Energies-Quantitative . . . . . 203

5.4.5 $\ddagger *$ Differential Cross-Section, $\mathrm{d} \sigma_{\text {in }} / \mathrm{d} E \ldots \ldots 204$

5.4.6 $\ddagger$ Partial and Total Cross-Sections, $\sigma_{\text {in }} \ldots \ldots$. . . . . 205

5.4.7 Quantification of EELS Core Edges . . . . . . . . . . 208

5.5 Energy-Filtered TEM Imaging (EFTEM) _ . . . . . . . . . . 209

5.5 .1 Spectrum Imaging _ . . . . . . . . . . . . . . . . 209

5.5 .2 Energy Filters . . . . . . . . . . . . . . . . . . . 210

5.5.3 Chemical Mapping with Energy-Filtered Images _ . . . 212

5.5.4 Chemical Analysis with High Spatial Resolution . . . . . 214

5.6 Energy Dispersive X-Ray Spectrometry (EDS) . . . . . . . . 216

5.6.1 Electron Trajectories Through Materials . . . . . . . . 216

5.6 .2 Fluorescence Yield . . . . . . . . . . . . . . . . . . . 219

5.6.3 EDS Instrumentation Considerations . . . . . . . . . . . 221

5.6.4 Artifacts in EDS Measurements . . . . . . . . . . . . 224

5.7 Quantitative EDS . . . . . . . . . . . . . . 225

5.7.1 Thin-Film Approximation . . . . . . . . . . . . 225

$5.7 .2 *$ ZAF Correction . . . . . . . . . . . . . 228

5.7.3 * Limits of Microanalysis . . . . . . . . . . . . . . . . 230

5.8 Further Reading . . . . . . . . . . . . . . . . . . . . . . . . 232

5.9 Problems . . . . . . . . . . . . . . . . . 233

6 Diffraction from Crystals . . . . . . . . . . . . . . 237

6.1 Sums of Wavelets from Atoms . . . . . . . . . . . . . 237

6.1.1 Electron Diffraction from a Material . . . . . . . . . . 238

6.1.2 Wave Diffraction from a Material . . . . . . . . . . . 240

6.2 The Reciprocal Lattice and the Laue Condition . . . . . . . . . 244

6.2.1 Diffraction from a Simple Lattice . . . . . . . . . . . . . 244

6.2 .2 Reciprocal Lattice . . . . . . . . . . . . . . . . . . . . 245

6.2 .3 Laue Condition . . . . . . . . . . . . . . . . . . . . 247

6.2.4 Equivalence of the Laue Condition and Bragg's Law . . . 247

6.2.5 Reciprocal Lattices of Cubic Crystals . . . . . . . . . . 248

6.3 Diffraction from a Lattice with a Basis . . . . . . . . . . . . . . 249

6.3.1 Structure Factor and Shape Factor . . . . . . . . . . . . 249

6.3.2 Structure Factor Rules . . . . . . . . . . . . . . . . 251

6.3.3 Symmetry Operations and Forbidden Diffractions . . . . . 256 
6.4 Chemically-Ordered Structures . . . . . . . . . . . . . . . 258

6.4.1 Superlattice Diffractions . . . . . . . . . . . . 258

6.4 .2 Order Parameters . . . . . . . . . . . . . . . . 261

6.5 Crystal Shape Factor . . . . . . . . . . . . . . . . . . . . 264

6.5.1 Shape Factor of Rectangular Prism . . . . . . . . . . . . . 264

6.5 .2 Other Shape Factors . . . . . . . . . . . . . . . . . . . . 268

6.5.3 Small Particles in a Large Matrix . . . . . . . . . . . . . . 269

6.6 Deviation Vector (Deviation Parameter) . . . . . . . . . . . . 272

6.7 Ewald Sphere . . . . . . . . . . . . . . . . . . . . 274

6.7.1 Ewald Sphere Construction . . . . . . . . . . . . . . 274

6.7.2 Ewald Sphere and Bragg's Law . . . . . . . . . . . . . . 275

6.7.3 Tilting Specimens and Tilting Electron Beams . . . . . . . 276

6.8 Laue Zones . . . . . . . . . . . . . . . . . . . . . . . . 278

$6.9 *$ Effects of Curvature of the Ewald Sphere . . . . . . . . . . . . 280

6.10 Further Reading . . . . . . . . . . . . . . . . . . . . 281

6.11 Problems ....................... 282

7 Electron Diffraction and Crystallography . . . . . . . . . . . . . . . 289

7.1 Indexing Diffraction Patterns . . . . . . . . . . . . . . . . . . 289

7.1 .1 Issues in Indexing . . . . . . . . . . . . . . . . . . . . . . . . . . . . . . . . . . . . .

7.1.2 Method 1-Start with Zone Axis . . . . . . . . . . . . . . 292

7.1.3 Method 2-Start with Diffraction Spots . . . . . . . . . . 296

7.2 Stereographic Projections and Their Manipulation . . . . . . . . . . 298

7.2.1 Construction of a Stereographic Projection . . . . . . . . . 298

7.2.2 Relationship Between Stereographic Projections and Electron Diffraction Patterns . . . . . . . . . . . . . 299

7.2.3 Manipulations of Stereographic Projections . . . . . . . . 300

7.3 Kikuchi Lines and Specimen Orientation . . . . . . . . . . . . . . . . . . . . 306

7.3.1 Origin of Kikuchi Lines . . . . . . . . . . . . . . . . . . . . . . . . . . . . . 306

7.3.2 Indexing Kikuchi Lines . . . . . . . . . . . . . . . . . 309

7.3.3 Specimen Orientation and Deviation Parameter . . . . . 311

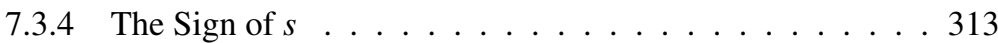

7.3.5 Kikuchi Maps . . . . . . . . . . . . . . . 314

7.4 Double Diffraction . . . . . . . . . . . . . . . 316

7.4.1 Occurrence of Forbidden Diffractions . . . . . . . . . . 316

7.4.2 Interactions Between Crystallites . . . . . . . . . . . 317

7.5* Convergent-Beam Electron Diffraction . . . . . . . . . . . . . . 318

7.5.1 Convergence Angle of Incident Electron Beam . . . . . . 319

7.5.2 Determination of Sample Thickness . . . . . . . . . . . 320

7.5.3 Measurements of Unit Cell Parameters . . . . . . . . . . . 322

7.5.4 $\ddagger$ Determination of Point Groups . . . . . . . . . . . . . . 328

7.5.5 $\ddagger$ Determination of Space Groups . . . . . . . . . . . . 339

7.6 Further Reading . . . . . . . . . . . . . . . . . . . . . . . . 342

7.7 Problems ........................ 343 
8 Diffraction Contrast in TEM Images . . . . . . . . . . . . . . . . . 349

8.1 Contrast in TEM Images . . . . . . . . . . . . . . . . . . . . 349

8.2 Diffraction from Crystals with Defects . . . . . . . . . . . 351

8.2.1 Review of the Deviation Parameter, $s \quad \ldots \ldots 351$

8.2.2 Atom Displacements, $\delta$ r . . . . . . . . . . . . . 352

8.2 .3 Shape Factor and $t \ldots \ldots 353$

8.2.4 Diffraction Contrast and $\{s, \boldsymbol{\delta} \boldsymbol{r}, t\} \ldots \ldots 353$

8.3 Extinction Distance . . . . . . . . . . . . . . . . . 354

8.4 The Phase-Amplitude Diagram . . . . . . . . . . . . . . . 356

8.5 Fringes from Sample Thickness Variations . . . . . . . . . . . 358

8.5.1 Thickness and Phase-Amplitude Diagrams . . . . . . . . 358

8.5.2 Thickness Fringes in TEM Images . . . . . . . . . . . 360

8.6 Bend Contours in TEM Images . . . . . . . . . . . . . . . . . . 363

8.7 Diffraction Contrast from Strain Fields . . . . . . . . . . . . . 368

8.8 Dislocations and Burgers Vector Determination . . . . . . . . . . 370

8.8.1 Diffraction Contrast from Dislocation Strain Fields . . . . 370

8.8.2 The $\boldsymbol{g} \cdot \boldsymbol{b}$ Rule for Null Contrast . . . . . . . . . . . . 372

8.8.3 Image Position and Dislocation Pairs or Loops . . . . . . 377

8.9 Semi-Quantitative Diffraction Contrast from Dislocations . . . . . 381

8.10 Weak-Beam Dark-Field (WBDF) Imaging of Dislocations . . . . 387

8.10 .1 Procedure to Make a WBDF Image . . . . . . . . . . 387

8.10 .2 Diffraction Condition for a WBDF Image . . . . . . . . 388

8.10 .3 Analysis of WBDF Images . . . . . . . . . . . . . . . 389

8.11 Fringes at Interfaces . . . . . . . . . . . . . . . . . . 393

8.11.1 Phase Shifts of Electron Wavelets Across Interfaces . . . . 393

8.11 .2 Moiré Fringes . . . . . . . . . . . . . . . . . . 396

8.12 Diffraction Contrast from Stacking Faults . . . . . . . . . . 400

8.12 .1 Kinematical Treatment . . . . . . . . . . . . . . . . . 400

8.12 .2 Results from Dynamical Theory . . . . . . . . . . . . 404

8.12.3 Determination of the Intrinsic or Extrinsic Nature of Stacking Faults . . . . . . . . . . . . . . 406

8.12.4 Partial Dislocations Bounding the Fault . . . . . . . . 407

8.12.5 An Example of a Stacking Fault Analysis . . . . . . . . 407

8.12.6 Sets of Stacking Faults in TEM Images . . . . . . . . . 409

8.12 .7 Related Fringe Contrast . . . . . . . . . . . . . . . . . . . 410

8.13 Antiphase $(\pi)$ Boundaries and $\delta$ Boundaries . . . . . . . . . 411

8.13 .1 Antiphase Boundaries . . . . . . . . . . . . . . . . . 411

$8.13 .2 \delta$ Boundaries . . . . . . . . . . . . . . . 412

8.14 Contrast from Precipitates and Other Defects . . . . . . . . 414

8.14 .1 Vacancies . . . . . . . . . . . . . . . . . . . . 414

8.14 .2 Coherent Precipitates . . . . . . . . . . . . . . . 415

8.14.3 Semicoherent and Incoherent Particles . . . . . . . . . . 419

8.15 Further Reading . . . . . . . . . . . . . . . . . . . . . . . . . . 420

8.16 Problems . . . . . . . . . . . . . . . . . 420 
9 Diffraction Lineshapes . . . . . . . . . . . . . . . . . . . . . . . 429

9.1 Diffraction Line Broadening and Convolution . . . . . . . . . . 429

9.1.1 Crystallite Size Broadening . . . . . . . . . . . . . . . 430

9.1 .2 Strain Broadening . . . . . . . . . . . . . . . . . 432

9.1.3 Instrumental Broadening-Convolution . . . . . . . . 436

9.2 Fourier Transform Deconvolutions . . . . . . . . . . . . . . 439

9.2.1 Mathematical Features . . . . . . . . . . . . . . . . . 439

9.2.2* Effects of Noise on Fourier Transform Deconvolutions . 442

9.3 Simultaneous Strain and Size Broadening . . . . . . . . . . . 446

9.4 Diffraction Lineshapes from Columns of Crystals . . . . . . . 452

9.4.1 Wavelets from Pairs of Unit Cells in One Column . . . . . 452

9.4.2 A Column Length Distribution . . . . . . . . . . . . . 454

9.4.3 ‡ Intensity from Column Length Distribution . . . . . . 456

9.5 Comments on Diffraction Lineshapes . . . . . . . . . . . . 458

9.6 Further Reading . . . . . . . . . . . . . . . . . . . . . . . 460

9.7 Problems . . . . . . . . . . . . . . . . . . . 460

10 Patterson Functions and Diffuse Scattering . . . . . . . . . . . 463

10.1 The Patterson Function . . . . . . . . . . . . . . 463

10.1.1 Overview . . . . . . . . . . . . . . . 463

10.1.2 Atom Centers at Points in Space . . . . . . . . . . . 464

10.1.3 Definition of the Patterson Function . . . . . . . . . . 465

10.1.4 Properties of Patterson Functions . . . . . . . . . . . . 467

10.1.5 $\ddagger$ Perfect Crystals . . . . . . . . . . . . . . . . . . 469

10.1.6 Deviations from Periodicity and Diffuse Scattering . . . . 473

10.2 Diffuse Scattering from Atomic Displacements . . . . . . . . 475

10.2.1 Uncorrelated Displacements—Homogeneous Disorder . . 475

10.2.2 $\neq$ Temperature . . . . . . . . . . . . . . . . 477

10.2.3 * Correlated Displacements-Atomic Size Effects . . . . . 482

10.3 Diffuse Scattering from Chemical Disorder . . . . . . . . . . 486

10.3.1 Uncorrelated Chemical Disorder-Random Alloys . . . . 486

10.3.2 ** SRO Parameters . . . . . . . . . . . . . . . . 490

10.3.3 $\$ *$ Patterson Function for Chemical SRO . . . . . . . 491

10.3.4 SRO Diffuse Intensity . . . . . . . . . . . . . . . . . 493

10.3.5 $\neq *$ Isotropic Materials . . . . . . . . . . . . . . . . . 493

10.3.6 * Polycrystalline Average and Single Crystal SRO . . . . . 495

$10.4 *$ Amorphous Materials . . . . . . . . . . . . . . . . . . 496

10.4.1 $\ddagger$ One-Dimensional Model . . . . . . . . . . . . . . . 496

10.4.2 $\neq$ Radial Distribution Function . . . . . . . . . . . 501

10.4.3 † Partial Pair Correlation Functions _. . . . . . . . 504

10.5 Small Angle Scattering . . . . . . . . . . . . . . . . . . . 506

10.5.1 Concept of Small Angle Scattering . . . . . . . . . . . . . 506

10.5.2* Guinier Approximation $($ Small $\Delta k) \ldots \ldots 08$

10.5.3 * Porod Law (Large $\Delta k) \ldots \ldots$. . . . . . . . . 512

10.5.4 $\neq *$ Density-Density Correlations (All $\Delta k$ ) . . . . . . . 514 
10.6 Further Reading . . . . . . . . . . . . . . . . . . . . . 516

10.7 Problems . . . . . . . . . . . . . . . 517

11 High-Resolution TEM Imaging . . . . . . . . . . . . . . . . . . 521

11.1 Huygens Principle . . . . . . . . . . . . . . . . 522

11.1.1 Wavelets from Points in a Continuum . . . . . . . . . 522

11.1.2 Huygens Principle for a Spherical Wavefront-Fresnel Zones ................... 526

11.1.3 $\ddagger$ Fresnel Diffraction Near an Edge . . . . . . . . . . . . 531

11.2 Physical Optics of High-Resolution Imaging . . . . . . . . . . . . 535

11.2.1 $\neq$ Wavefronts and Fresnel Propagator . . . . . . . . . . . . 535

$11.2 .2 \ddagger$ Lenses . . . . . . . . . . . . . . . . . 537

$11.2 .3 \ddagger$ Materials . . . . . . . . . . . . . . . . 539

11.3 Experimental High-Resolution Imaging . . . . . . . . . . . . . . . . . . . 542

11.3.1 Defocus and Spherical Aberration . . . . . . . . . . . . . 542

11.3.2 † Lenses and Specimens . . . . . . . . . . . . . . . . . . . . . . . . 546

11.3.3 Lens Characteristics . . . . . . . . . . . . . . . . 550

$11.4 *$ Simulations of High-Resolution TEM Images . . . . . . . . . 557

11.4.1 Principles of Simulations . . . . . . . . . . . . . . . 558

11.4.2 Practice of Simulations . . . . . . . . . . . . . 563

11.5 Issues and Examples in High-Resolution TEM Imaging . . . . . . 564

11.5.1 Images of Nanostructures . . . . . . . . . . . . . . . . . . 564

11.5.2 Examples of Interfaces . . . . . . . . . . . . . . . 567

11.5.3* Specimen and Microscope Parameters . . . . . . . . . 570

11.5.4* Some Practical Issues for HRTEM . . . . . . . . . . . 577

11.5.5* Geometric Phase Analysis . . . . . . . . . . . . . . 580

11.6 Further Reading . . . . . . . . . . . . . . . . . . . . . . . . . . . . . . . . . . . . . . . . . . . . . . . . . .

11.7 Problems . . . . . . . . . . . . . . . . . . 584

12 High-Resolution STEM and Related Imaging Techniques . . . . . 587

12.1 Characteristics of High-Angle Annular Dark-Field Imaging . . . . 587

12.2 Electron Channeling Along Atomic Columns . . . . . . . . . . . 590

12.2.1 Optical Fiber Analogy . . . . . . . . . . . . . . . . 590

12.2.2 $\neq$ Critical Angle . . . . . . . . . . . . . . . . 592

12.2.3* Tunneling Between Columns . . . . . . . . . . . . 593

12.3 Scattering of Channeled Electrons . . . . . . . . . . . . . . . 595

12.3.1 Elastic Scattering of Channeled Electrons . . . . . . . . 595

12.3.2* Inelastic Scattering of Channeled Electrons . . . . . . . 597

$12.4 *$ Comparison of HAADF and HRTEM Imaging . . . . . . . . . . 598

12.5 HAADF Imaging with Atomic Resolution . . . . . . . . . . . . . . . . . . . 599

12.5.1* Effect of Defocus . . . . . . . . . . . . . . . 599

12.5.2 Experimental Examples . . . . . . . . . . . . . . . 601

$12.6 *$ Lens Aberrations and Their Corrections . . . . . . . . . . . . . 602

12.6.1 $C_{\mathrm{s}}$ Correction with Magnetic Hexapoles . . . . . . . . . . 602

12.6.2 $\ddagger$ Higher-Order Aberrations and Instabilities . . . . . . . . 605

12.7 Examples of $C_{\mathrm{s}}$-Corrected Images . . . . . . . . . . . . . 607 
12.7.1 Three-Dimensional Imaging . . . . . . . . . . . 608

12.7.2 High Resolution EELS . . . . . . . . . . . . . . . 610

12.8 Electron Tomography . . . . . . . . . . . . . . . . . . 610

12.9 Further Reading . . . . . . . . . . . . . . . . . . . . . . . 614

12.10 Problems . . . . . . . . . . . . . . . . 614

13 Dynamical Theory . . . . . . . . . . . . . . . . . 6617

13.1 Chapter Overview . . . . . . . . . . . . . . . . . . . 617

$13.2 \ddagger *$ Mathematical Features of High-Energy Electrons

in a Periodic Potential . . . . . . . . . . . . . . . . . . . . 619

13.2.1 $\neq *$ The Schrödinger Equation . . . . . . . . . . . . 619

13.2.2 $\$$ Kinematical and Dynamical Theory . . . . . . . . . . 625

13.2.3 * The Crystal as a Phase Grating . . . . . . . . . . . . . 627

13.3 First Approach to Dynamical Theory-Beam Propagation . . . . 629

$13.4+$ Second Approach to Dynamical Theory-Bloch Waves and Dispersion Surfaces . . . . . . . . . . . . . 633

13.4.1 Diffracted Beams, $\left\{\Phi_{g}\right\}$, are Beats of Bloch Waves, $\left\{\Psi^{(j)}\right\} 633$

13.4.2 Crystal Periodicity and Dispersion Surfaces . . . . . . . . 639

13.4.3 Energies of Bloch Waves in a Periodic Potential . . . . . . 642

13.4.4 General Two-Beam Dynamical Theory . . . . . . . . . . . 645

13.5 Essential Difference Between Kinematical and Dynamical

Theories . . . . . . . . . . . . . . . . 652

$13.6 \ddagger$ Diffraction Error, $s_{\boldsymbol{g}}$, in Two-Beam Dynamical Theory . . . . . 655

13.6.1 Bloch Wave Amplitudes and Diffraction Error . . . . . . . 655

13.6.2 Dispersion Surface Construction . . . . . . . . . . . 658

13.7 Dynamical Diffraction Contrast from Crystal Defects . . . . . . . 660

13.7.1 Dynamical Diffraction Contrast Without Absorption . . . 660

13.7.2 $\neq *$ Two-Beam Dynamical Theory of Stacking Fault

Contrast .................. 665

13.7.3 Dynamical Diffraction Contrast with Absorption . . . . . 668

$13.8 \ddagger *$ Multi-Beam Dynamical Theories of Electron Diffraction . . . 673

13.9 Further Reading . . . . . . . . . . . . . . . . . . . . 676 . . . . . . 676

13.10 Problems ...................... 676

Appendix ......................... 681

A.1 Indexed Powder Diffraction Patterns . . . . . . . . . . . . 681

A.2 Mass Attenuation Coefficients for Characteristic $K \bar{\alpha}$ X-Rays . . . 681

A.3 Atomic Form Factors for X-Rays . . . . . . . . . . . . . . . 683

A.4 X-Ray Dispersion Corrections for Anomalous Scattering . . . . . 683

A.5 Atomic Form Factors for $200 \mathrm{keV}$ Electrons and Procedure

for Conversion to Other Voltages . . . . . . . . . . . . . . . . . . 689

A.6 Indexed Single Crystal Diffraction Patterns: fcc, bcc, dc, hcp . . . 695

A.7 Stereographic Projections . . . . . . . . . . . . . 705

A.8 Examples of Fourier Transforms . . . . . . . . . . . . . . . . . 709

A.9 Debye-Waller Factor from Wave Amplitude . . . . . . . . . . . . 711

A.10 Time-Varying Potentials and Inelastic Neutron Scattering . . . . . 712 
A.11 Review of Dislocations . . . . . . . . . . . . . . . 715

A.12 TEM Laboratory Exercises . . . . . . . . . . . . . . . 721

A.12.1 Laboratory 1 -Microscope Procedures and Calibration with $\mathrm{Au}$ and $\mathrm{MoO}_{3} \ldots \ldots \ldots$. . . . . . . . . 721

A.12.2 Laboratory 2-Diffraction Analysis of $\theta^{\prime}$ Precipitates . . . 725

A.12.3 Laboratory 3-Chemical Analysis of $\theta^{\prime}$ Precipitates . . . . 728

A.12.4 Laboratory 4-Contrast Analysis of Defects . . . . . . . 729

A.13 Fundamental and Derived Constants . . . . . . . . . . . 730

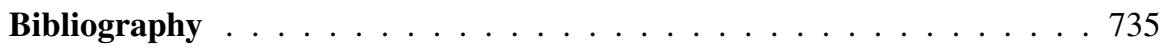

Further Reading . . . . . . . . . . . . . . . . . . . . 735

References and Figures . . . . . . . . . . . . . . . . . 739

Index . . . . . . . . . . . . . . . . . . . . . . . . 747 